\title{
Lichen planus pemphigoides: Lesional immunopathologic features
}

\author{
Ana Maria Abreu Velez', Billie L. Jackson², Michael S. Howard'1 \\ ${ }^{1}$ Georgia Dermatopathology Associates, Atlanta, Georgia, USA, ${ }^{2}$ Billie L. Jackson, M.D., Dermatologist, Macon, Georgia, \\ USA
}

Corresponding author: Dr. Ana Maria Abreu Velez, M.D., Ph.D., E-mail: abreuvelez@yahoo.com

\begin{abstract}
Lichen planus pemphigoides (LPP) is a rare autoimmune bullous dermatosis. The disease features combined features of bullous pemphigoid and lichen planus. Here we describe a case with histological and immunopathological features of LPP in a 59 year old male. Skin biopsies were taken for hematoxylin and eosin (H\&E) as well for direct immunofluorescence (DIF) and for immunohistochemistry (IHC) stains. The H\&E staining displayed a subepidermal blister, with a dense lymphohistiocytic infiltrate located along the basement membrane zone of the skin (BMZ). The DIF was positive at the BMZ for IgG, C3c, Clq, fibrinogen and albumin. The same markers were positive in a linear dermal band. Cytoid bodies were positive with IgG. The IHC staining demonstrated very positive staining over the entire BMZ with metallothionein, as well as C5b-9/MAC staining. The inflammatory infiltrate was positive for HLADPDQDR antigen. Our findings demonstrate that in this case HLA-DPDQDR antigen may play an important role presenting the antigens, and the roles of $\mathrm{C} 5 \mathrm{~b}-9 / \mathrm{MAC}$ and metallothionein need further investigation.
\end{abstract}

Key words: Lichen planus pemphigoides; Cytoid bodies; Basement membrane zone; Metallothionein

Abbreviations: Lichen planus pemphigoides (LPP), hematoxylin and eosin (H\&E), immunohistochemistry (IHC), direct immunofluorescence (DIF), basement membrane zone (BMZ), 4',6-diamidino-2-phenylindole (DAPI).

\section{INTRODUCTION}

Lichen planus pemphigoides (LPP) is a rare, autoimmune bullous dermatosis first described by Dr. Moritz Kaposi, and demonstrating combined features of lichen planus and bullous pemphigoid [1]. The clinical presentation of LPP may simulate bullous pemphigoid, making the diagnosis problematic. Clinically, LPP is characterized by a sudden onset of tense, dome-shaped bullae preceding, during, or after an eruption of lichen planus [1]. The blisters may arise on uninvolved skin or on pre-existing lichen planus lesions [2]. In LPP the lesions most usually involve the distal extremities, but they may occur in a generalized form. Oral and conjunctival mucosal involvement has been reported [3]. LPP more commonly affects males, usually in the fourth or fifth decade of life. LPP has been also associated with prescription and over the counter medications, including herbal supplements, angiotensin-converting- enzyme inhibitors, PUVA (including narrow band therapy), weight reduction products and simvastatin among others [4-6]. A detailed clinical, histopathological, and immunological evaluation is essential for the diagnosis of LPP. The basement membrane zone (BMZ) is considered one of the most affected structures in LPP. It is important to ask patients about any systemic disorders as well as current mediations. The etiology is fundamentally idiopathic; however, there are numerous case reports of drug-induced LPP.

\section{Statement of Ethics}

Our patient gave informed consent. Although Institutional Review Board (IRB) approval for a case report is not needed, the US Health Insurance

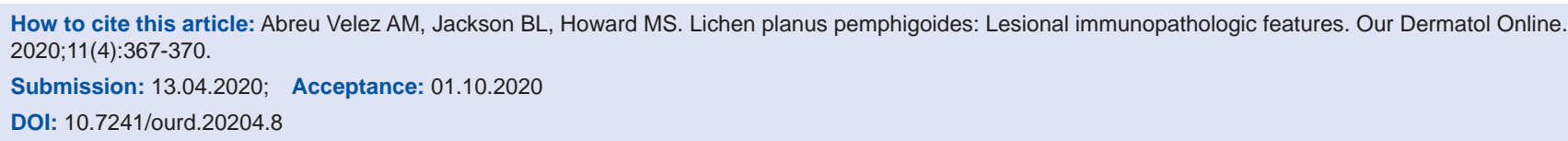


Portability and Accountability Act of 1996 (HIPAA) Privacy Rule restricts how protected health information (individually identifiable health information) on any patient may be made. Compliance with patient privacy, institutional rules, and federal regulations were followed. No photos or illustrations that contain identifiable features are included in the case report, and the case(s) described in the report are not so unique or unusual that it might be possible for others to identify the patients.

A 59 old male consulted the dermatologist for the presence of persistent patches with pruritic, pink to violaceous, flattopped, polygonal papules on the volar wrists, extensor elbows, and bilateral lower legs of 6 months duration. Some areas showed hyperpigmentation and scaling. The patient further develops some microvesicles. Systemically he had no significant medial history and was not taking medications. Skin biopsies were taken for hematoxylin and eosin (H\&E) as well for direct immunofluorescence (DIF) and for immunohistochemistry (IHC) staining. These were performing as previously described [7-9]. Workup revealed lichen planus pemphigoides (LPP). The histopathology and direct immunofluorescence were compatible with LPP. The patient was prescribed with Clobetasol propionate ${ }^{\mathrm{TM}}$ cream $0.05 \%$ for the trunk, extremities, and nails and momethasone furoate cream $0.1 \%$ for the face with improvement; anti-histamine was added for the itching.

Skin biopsies were taken for hematoxylin and eosin(H\&E) staining, direct immunofluorescence (DIF) and for immunohistochemistry (IHC) stains, which were performed as previously described [8-11]. For DIF, we classified our findings as previously categorized [8-11], i.e. negative (-), weakly positive $(+)$, positive $(+++)$ and strongly positive $(++++)$.

IHC stains were performed utilizing a Leica Bond MAX IHC automatized platform (Buffalo Grove, Illinois, USA) using Novolink ${ }^{\mathrm{TM}}$ detection with Compact Polymer ${ }^{\mathrm{TM}}$ technology. Specifically, for primary staining we utilized a Bond Max platform autostainer with bond polymer refined Red detection DS9390, an alkaline phosphatase linker polymer and fast red chromogen (red staining). For our secondary staining, we utilized bond polymer refined detection DS9800, a horseradish peroxidase linker polymer and DAB chromogen (brown staining). The staining was performed as previously described. Positive and negative controls were consistently performed. We used mouse antihuman monoclonal Dako (Carpinteria, California,
USA) antibodies to HLA-DPDQDR antigen (clone CR3/43), complement/C5b-9/MAC (clone aE11) and metallothionein (clone E9).

Examination of the H\&E tissue sections demonstrated a dense lymphohistiocytic infiltrate located along the dermal/epidermal junction. In addition, occasional Civatte body necrotic keratinocytes were present within these areas (Fig. 1). A subepidermal blister was seen. The results of the DIF are shown in Table 1 and are also shown in Figure 1.

The IHC showed strong positive staining along the entire BMZ with metallothionein, being stronger in

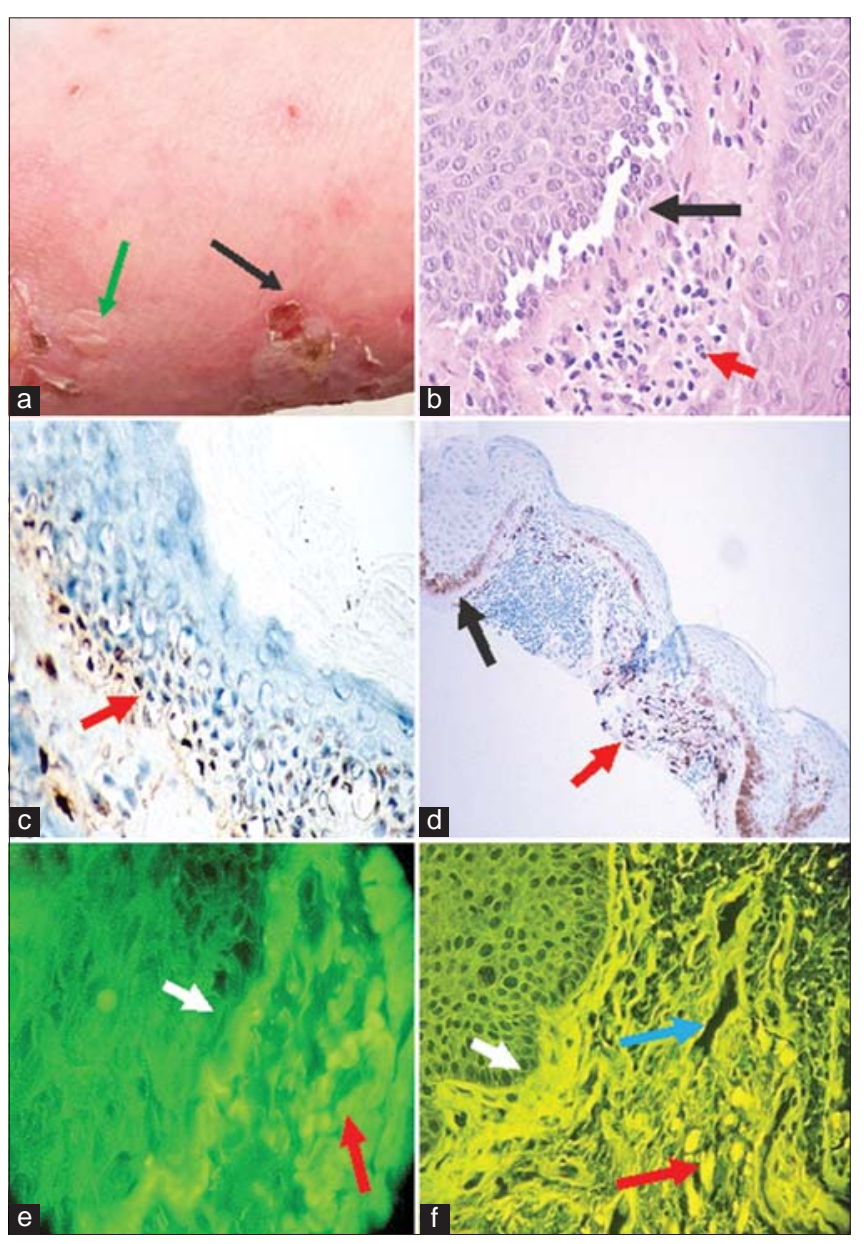

Figure 1: (a-b) H\&E staining at 200 and 400X respectively, showing some basal layer involvement with some basement membrane zone separation(black arrows) and an inflammatory infiltrate in the upper dermis (red arrows). c. IHC positive staining at the BMZ using C5b9/MAC (dark staining, red arrow; 400X). d. IHC showing positive staining with metallothionein at the BMZ (black arrow), but also in the upper dermal inflammatory infiltrate (red arrow; 100X). e. DIF positive staining at the BMZ (white arrow), as well as against the upper dermal inflammatory cells using FITC conjugated anti-human IgG (red arrow). f. DIF positive staining for IgG at the BMZ (white arrow), against upper dermal blood vessels (blue arrow) and involvement of individual cells including the presence of cytoid bodies(red arrow; 400X). 
Table 1: DIF Results

\begin{tabular}{|c|c|}
\hline Catalog no., antibody, dilution & Results \\
\hline $\begin{array}{l}\text { F0202-2.Polyclonal rabbit anti- } \\
\text { human IgG FITC, 1:20 dil, Agilent } \\
\text { Dako. }\end{array}$ & $\begin{array}{l}\text { Positive linear deposits at the BMZ } \\
\text { junction (+++), and also positive } \\
\text { clumps in the superficial dermis, } \\
\text { resembling those seen in lichen } \\
\text { planus (+++.) }\end{array}$ \\
\hline $\begin{array}{l}\text { F0203-2. Polyclonal rabbit anti- } \\
\text { human IgM FITC, 1:20 dil, Agilent } \\
\text { Dako. }\end{array}$ & Negative \\
\hline $\begin{array}{l}\text { F0204-2.Polyclonal rabbit anti- } \\
\text { human IgA FITC, 1:20 dil, Agilent } \\
\text { Dako. }\end{array}$ & Negative \\
\hline $\begin{array}{l}\text { F020102-2.Polyclonal rabbit anti- } \\
\text { human C3 FITC, 1:20 dil, Agilent } \\
\text { Dako. }\end{array}$ & $\begin{array}{l}\text { Positive granular deposits at the } \\
\text { BMZ junction) }(+) \text {. }\end{array}$ \\
\hline $\begin{array}{l}\text { F0111-02. Polyclonal rabbit anti- } \\
\text { human Fibrinogen FITC, 1:40 dil, } \\
\text { Agilent Dako. }\end{array}$ & $\begin{array}{l}\text { Positive linear stain deposit at the } \\
\mathrm{BMZ}(++) \text {. }\end{array}$ \\
\hline $\begin{array}{l}\text { D2030-02. Anti-human IgD FITC, dil } \\
\text { 1:25, Southern Biotech. }\end{array}$ & $\begin{array}{l}\text { Positive linear deposits at the BMZ } \\
\text { junction (++), and also positive } \\
\text { clumps in the upper superficial } \\
\text { dermis, resembling those seen in } \\
\text { lichen planus (++). }\end{array}$ \\
\hline $\begin{array}{l}\text { F0117-2.Polyclonal rabbit anti- } \\
\text { human Albumin FITC 1:40 dil, } \\
\text { Agilent Dako }\end{array}$ & $\begin{array}{l}\text { Positive linear deposits at the BMZ } \\
(+++) \text {, and also positive clumps in } \\
\text { the superficial dermis, resembling } \\
\text { those seen in lichen planus }(+++) \text {. }\end{array}$ \\
\hline $\begin{array}{l}\text { SKU } 104202 \quad \text { Anti-human } \\
\text { IgE, FITC 1:25 dil, Low F/P - 2-4.9, } \\
\text { from Kent Laboratories. }\end{array}$ & Negative \\
\hline $\begin{array}{l}\text { F0254-2. Polyclonal rabbit anti- } \\
\text { human C1-q FITC, 1:20 dil, Agilent } \\
\text { Dako. }\end{array}$ & Negative \\
\hline Negative control & Negative \\
\hline
\end{tabular}

some areas of the BMZ and also strong positivity with C5b-9/MAC. We also observed positive staining in the dermal infiltrate with HLA-DPDQDR antigen.

\section{DISCUSSION}

It has been previously described that patients with classic lichen planus who later experience subepidermal blisters on the trunk and extremities are clinical features consistent with lichen planus pemphigoides [1-3]. Usually the histopathology of LPP is categorized by typical findings of a lichenoid tissue reaction with subepidermal bullae and linear deposits of $\operatorname{IgG}$ and $\mathrm{C} 3$ along the basement membrane zone on DIF of peri-bullous skin. Immunofluorescence of perilesional skin shows usually linear deposits of $\mathrm{C} 3$ along the dermoepidermal junction or BMZ [3]. The patient IgG autoantibodies have been shown to be directed to the $180 \mathrm{kD}$ bullous pemphigoid antigen (BPAG2, Type XVII collagen) using immunoblotting. Interestingly, we also detected positive patchy staining with metallothionein and C5b-9/MAC at the basement membrane zone of the skin. Our findings attest to the similarity of immunopathology in these two subepidermal blistering skin diseases. The pathogenesis of LPP is partly explained. Some authors hypothesize that a primary inflammatory process by lichen planus causes the release and exposure of other molecules that were not initially recognized as antigens by the immune system, possibly leading to epitope spreading and/or to a secondary autoimmune response against the BMZ. These circulating autoantibodies may then induce a secondary, subepidermal bullous dermatosis. Further, previous authors described the presence of LPP in a patient whom later presented with herpes gestationis [10]. In this case, the distension of the abdominal surface may have exposed new antigens.

LPP has been also associated with chronic hepatitis B virus infection, as documented in lichen planus [11].

We searched for studies that investigated mononuclear cells in an LPP infiltrate, and found very few studies. Here we include the best study and their results: mean percentage of positively stained dermal-infiltrating cells were shown to be a mix of CD8 and CD20, followed by minor percentages of CD3, CD4 and FOXP [12]. These authors noted that the cell infiltrate were a combination of cells previously documented in lesional areas of bullous pemphigoid and lichen planus, indicating that LPP is a unique nosologic entity.

Our DIF findings also show a clear mix of the pertinent disease features, with cytoid bodies of lichen planus, the BMZ involvement and the dermal band staining; our findings are similar to those described by others [13], with the addition of the strong immunofluorescence features in the dermal band and cytoid bodies.

In our case, we also note the lesional presence of C5b-9/MAC at the BMZ in this case of lichen planus pemphigoides. The presence of complement C5b-9/ MAC has also been recently detected in patients affected by endemic pemphigus foliaceus in El Bagre, Colombia, South America in skin biopsies correlating with disease severity and previously established serologies [14]. It would be of interest to study for the presence of C5b-9/MAC in a larger series of patients affected by this disease. Recently, several groups have shown the presence of metallothionein expression in lichen planus epithelial and inflammatory cells. These authors suggested an extensive anti-apoptotic response in the keratotic form of the disease [15]. Thus, the roles of C5-b9/MAC and metallothionein in LPP warrant further studies. 


\section{Consent}

The examination of the patient was conducted according to the Declaration of Helsinki principles.

The authors certify that they have obtained all appropriate patient consent forms. In the form the patient(s) has/have given his/her/ their consent for his/her/their images and other clinical information to be reported in the journal. The patients understand that their names and initials will not be published and due efforts will be made to conceal their identity, but anonymity cannot be guaranteed.

\section{REFERENCES}

1. Kaposi M. Lichen ruber pemphigoides. Arch J Dermatol Syph. 1892;24:343-46.

2. Tamada Y, Yokochi K, Nitta Y, Ikeya T, Hara K, Owaribe K. Lichen planus pemphigoides: identification of $180 \mathrm{kd}$ hemidesmosome antigen. J Am Acad Dermatol. 1995;32:883-87.

3. Zaraa I, Mahfoudh A, Sellami MK, Chelly I, El Euch D, Zitouna M, et al. Lichen planus pemphigoides: four new cases and a review of the literature. Int J Dermatol. 2013;52:406-12.

4. Flageul B, Foldes C, Wallach D, Vignon-Pennamen MD, Cottenot F. Captopril-induced lichen planus pemphigoides with pemphigus-like features. A case report. Dermatologica. 1986;173:248-55.

5. Mandy Chan WM, Lee JS, Thiam Theng CS, Chua SH, Boon Oon HH. Narrowband UVB-induced lichen planus pemphigoide. Dermatol Reports. 2011;3:e43.

6. Xu HH, Xiao T, He CD, Jin GY, Wang YK, Gao XH, et al. Lichen planus pemphigoides associated with Chinese herbs. Clin Exp Dermatol. 2009;34:329-32.

7. Abreu Velez AM, Upegui Zapata YA, Howard MS. Periodic Acid-Schiff staining parallels the immunoreactivity seen by direct immunofluorescence in autoimmune skin diseases. N Am J Med
Sci. 2016;8:151-5.

8. Abreu-Velez AM, Googe PB, Howard MS. Immunohistochemistry versus immunofluoresence in the diagnosis of autoimmune blistering diseases. Our Dermatol Online. 2013;4-3S:585-95.

9. Abreu-Velez AM, Vasquez-Hincapie DA, Howard MS. Autoimmune basement membrane and subepidermal blistering diseases. Our Dermatol Online. 2013;4(Suppl.3):647-62.

10. Rullán J, Diaz NC, Vazquez-Botet M. Lichen planus pemphigoides associated with pregnancy mimicking pemphigoid gestationis. Cutis. 2016;97:E20-4.

11. Kabuto M, Fujimoto N, Yamaguchi A, Tanaka T. Evaluation of mononuclear cells in lichen planus pemphigoides. Acta Derm Venereol. 2016;96:276-8.

12. Jang SH, Yun SJ, Lee SC, Lee JB. Lichen planus pemphigoides associated with chronic hepatitis B virus infection. Clin Exp Dermatol. 2015;40:868-71.

13. Arbache ST, Nogueira TG, Delgado L, Miyamoto D, Aoki V. Immunofluorescence testing in the diagnosis of autoimmune blistering diseases: overview of 10-year experience. An Bras Dermatol. 2014;89:885-9.

14. Abreu-Velez AM, Upegui-Zapata YA, Valencia-Yepes CA, UpeguiQuiceno E, Mesa-Herrera NR, Jiménez-Echavarria AM, et al. Membrane attack complex (C5b-9 complex or MAC), is strongly present in lesional skin from patients with endemic pemphigus foliaceus in El Bagre, Colombia. J Cutan Pathol. 2019;46:925-99.

15. Allon I, Ofir M, Vered H, Hirshberg A. Metallothionein, a marker of antiapoptosis, is associated with clinical forms of oral lichen planus. J Oral Pathol Med. 2014;43:728-33.

Copyright by Ana Maria Abreu Velez, et al. This is an open-access article distributed under the terms of the Creative Commons Attribution License, which permits unrestricted use, distribution, and reproduction in any medium, provided the original author and source are credited.

Source of Support: Nil, Conflict of Interest: Georgia Dermatopathology Associates (GDA). 